\title{
Atheroprotection through SYK inhibition fails in established disease when local macrophage proliferation dominates lesion progression
}

\author{
Alexandra Lindau ${ }^{1}$ - Carmen Härdtner ${ }^{1} \cdot$ Sonja P. Hergeth ${ }^{1} \cdot$ Kelly Daryll Blanz $^{1,2,3}$. \\ Bianca Dufner $^{1} \cdot$ Natalie Hoppe $^{1} \cdot$ Nathaly Anto-Michel $^{1} \cdot$ Jan Kornemann $^{1}$ \\ Jiadai Zou ${ }^{1} \cdot$ Louisa M. S. Gerhardt ${ }^{1}$. Timo Heidt ${ }^{1}$ - Florian Willecke ${ }^{1}$. \\ Serjosha Geis ${ }^{1}$ - Peter Stachon ${ }^{1}$ - Dennis Wolf ${ }^{1} \cdot$ Peter Libby $^{4} \cdot$ Filip K. Swirski $^{5}$. \\ Clinton S. Robbins ${ }^{6} \cdot$ William McPheat $^{7} \cdot$ Shaun Hawley $^{8} \cdot$ Martin Braddock $^{8}$. \\ Ralf Gilsbach ${ }^{9} \cdot$ Lutz Hein $^{9}$ - Constantin von zur Mühlen ${ }^{1}$ Christoph Bode ${ }^{1}$ • \\ Andreas Zirlik ${ }^{1} \cdot$ Ingo Hilgendorf $^{1}$
}

Received: 10 August 2015/Accepted: 21 January 2016/Published online: 18 February 2016

(C) The Author(s) 2016. This article is published with open access at Springerlink.com

\begin{abstract}
Macrophages in the arterial intima sustain chronic inflammation during atherogenesis. Under hypercholesterolemic conditions murine Ly6 $\mathrm{C}^{\text {high }}$ monocytes surge in the blood and spleen, infiltrate nascent atherosclerotic plaques, and differentiate into macrophages that proliferate locally as disease progresses. Spleen tyrosine kinase (SYK) may participate in downstream signaling of various receptors that mediate these processes. We tested the effect of the SYK inhibitor fostamatinib on hypercholesterolemia-associated myelopoiesis and plaque formation in $\mathrm{Apoe}^{-/-}$mice during early and established atherosclerosis. Mice consuming a high cholesterol diet supplemented with fostamatinib for 8 weeks developed less atherosclerosis. Histologic and flow cytometric
\end{abstract}

A. Lindau, C. Härdtner, A. Zirlik and I. Hilgendorf contributed equally.

Electronic supplementary material The online version of this article (doi:10.1007/s00395-016-0535-8) contains supplementary material, which is available to authorized users.

Andreas Zirlik

andreas.zirlik@universitaets-herzzentrum.de

$\triangle$ Ingo Hilgendorf

ingo.hilgendorf@universitaets-herzzentrum.de

1 Department of Cardiology and Angiology I, University Heart Center Freiburg, Hugstetter Str. 55, 79106 Freiburg, Germany

2 Spemann Graduate School of Biology and Medicine, University of Freiburg, Freiburg, Germany

3 Faculty of Biology, University of Freiburg, Freiburg, Germany analysis of aortic tissue showed that fostamatinib reduced the content of Ly6C ${ }^{\text {high }}$ monocytes and macrophages. SYK inhibition limited Ly6 $\mathrm{C}^{\text {high }}$ monocytosis through interference with GM-CSF/IL-3 stimulated myelopoiesis, attenuated cell adhesion to the intimal surface, and blocked M-CSF stimulated monocyte to macrophage differentiation. In $\mathrm{Apoe}^{-/-}$mice with established atherosclerosis, however, fostamatinib treatment did not limit macrophage accumulation or lesion progression despite a significant reduction in blood monocyte counts, as lesional macrophages continued to proliferate. Thus, inhibition of hypercholesterolemia-associated monocytosis, monocyte infiltration, and differentiation by SYK antagonism attenuates early atherogenesis but not established disease when local macrophage proliferation dominates lesion progression.

Keywords Atherosclerosis - SYK - Monocytes · Macrophages · Progenitors · Proliferation · Egress

4 Department of Medicine, Brigham and Women's Hospital, Harvard Medical School, Boston, MA, USA

5 Center for Systems Biology, Massachusetts General Hospital, Harvard Medical School, Boston, MA, USA

6 Peter Munk Cardiac Centre, Toronto, ON, Canada

7 AstraZeneca R\&D, Molndal, Gothenburg, Sweden

8 AstraZeneca R\&D, Alderley Park, Macclesfield, UK

9 Institute of Experimental and Clinical Pharmacology and Toxicology, University of Freiburg, Freiburg, Germany 


\section{Introduction}

Atherosclerosis involves the accumulation of lipids and inflammatory cells in the arterial intima forming plaques. Macrophages ingest lipids and elaborate inflammatory mediators that promote plaque progression and destabilization [1]. During atheroma initiation, macrophages accumulate primarily through infiltration of Ly $6 \mathrm{C}^{\text {high }}$ monocytes. In established plaques, however, macrophages renew through local proliferation rather than recruitment [13]. It is unknown whether prolonged interference with monocyte recruitment to established plaques can limit macrophage accumulation.

Spleen tyrosine kinase (SYK), a cytosolic signaling protein, binds to various receptors and adaptors containing immunoreceptor tyrosine-based activation motifs (ITAM). Integrin signaling in myeloid cells, for example, requires the ITAM-bearing DAP12 adaptor and subsequent SYK activation to induce cell adhesion $[9,16]$. We showed that treatment with the orally available SYK inhibitor fostamatinib reduced inflammatory cell recruitment to the vessel wall and infiltration to sites of inflammation in low density lipoprotein receptor deficient $\left(\mathrm{Ldlr}^{-/-}\right)$mice. Consequently, these animals developed less atherosclerosis [5]. Of note, fostamatinib demonstrated significant improvement in ACR20 scores over placebo in two recent phase 3 trials with rheumatoid arthritis (RA) patients [5, 17]. The long-term effects of this SYK inhibitor on cardiovascular events in this high risk study population with chronic inflammation and accelerated atherosclerosis [4, 14] remains unknown.

The present study compared the efficacy of SYK inhibition in early and established atherosclerosis. We used $\mathrm{Apoe}^{-/-}$mice, which develop highly inflammatory atherosclerosis characterized by hypercholesterolemia-associated monocytosis [15]. The myelopoietic growth factors GM-CSF and IL-3 stimulate monocyte production in atherosclerosis [12]. Their receptors share a common beta chain (CBS) that associates with SYK [18]. We hypothesized that SYK inhibition by fostamatinib would reduce monocytosis and cell adhesion, and provide us with a tool to lower monocyte contribution to the plaque in early and late atherosclerosis.

\section{Methods}

\section{Animal models}

6-week-old female Apoe ${ }^{-/-}$mice (B6.129P2-Apoe ${ }^{\text {tm1Unc }}$ / J) were purchased from Charles River (Calco, Italy) and consumed a high cholesterol diet $(1.25 \%$ cholesterol; D12108 mod., Ssniff GmBH, Soest, Germany) ad libitum for 8 and 20 weeks, respectively, as indicated. Fostamatinib disodium (Astra Zeneca, UK) without carrier was incorporated at $0.3 \%(\mathrm{w} / \mathrm{w})$ into the diet as previously described [6]. $1 \mathrm{mg}$ BrdU per mouse (BD Bioscience, San Jose, CA, USA) was injected intravenously $2 \mathrm{~h}$ before euthanasia. Intravital microscopy of mesenteric veins was performed $3 \mathrm{~h}$ after intraperitoneal stimulation with TNF $\alpha$. Ly6 $\mathrm{C}^{\text {high }}$ monocytes were mobilized into peripheral blood by intravenous injection of $300 \mathrm{ng}$ CCL2 (R\&D System, Minneapolis, MN, USA). $1 \mu \mathrm{m}$ large green fluorescent beads (Fluoresbrite YG plain microspheres, Polysciences Inc., Eppelheim, Germany), diluted 1:4 in sterile PBS, were injected intravenously for in vivo cell labeling and tracking. Mice were housed under specific pathogen-free conditions and procedures approved by the Animal Care Committee of the University of Freiburg and the Regional Council (G-12/083).

\section{Histology}

Aortic roots were embedded in OCT Tissue Tek (Sakura Finetek, Tokyo, Japan) for sectioning and staining with specific antibodies that are listed in the supplemental method section. Abdominal aortas were pinned for Oli-red $\mathrm{O}$ en face staining.

\section{Flow cytometry and cell sorting}

Cells isolated from the blood, spleen, bone marrow and aorta were stained with specific antibodies and dyes that are listed in the supplemental method section. The APC Brdu Flow Kit detected intracellular BrdU incorporation (BD Bioscience, San Diego, CA, USA) according to the manufacturer's instructions. Intracellular phospho-SYKTyr519/520 (Cell Signaling Technology, Boston, MA, USA) was evaluated in fixed and permeabilized (ebioscience, San Jose, CA, USA) bone marrow and blood cells. Data were recorded on a BD Facs Canto II (BD Bioscience, San Diego, CA, USA). Monocytes and macrophages were sorted with a BD Facs Aria III (BD Bioscience, San Diego, CA, USA).

\section{Colony forming cell assay}

Bone marrow cells were isolated from the femur and resuspended in Mouse Methylcellulose Complete Medium (R\&D, Minneapolis, MN, USA) at $10^{5}$ cells $/ \mathrm{ml}$. Cells were stimulated with $10 \mathrm{ng} / \mathrm{ml}$ GM-CSF and $10 \mathrm{ng} / \mathrm{ml}$ IL3 (Peprotech, Rocky Hill, NJ, USA) in the presence of the vehicle DMSO 0.1\%, 0.1uM R406 in DMSO $0.1 \%$ and $1 \mu \mathrm{M}$ R406 in $0.1 \%$ DMSO, respectively, as indicated. Cells were incubated at $37{ }^{\circ} \mathrm{C}$ and $5 \% \mathrm{CO}_{2}$ for 5 days according to the manufacturer's instructions and the 
number of colonies per $35 \mathrm{~mm}$ culture plate was counted under the microscope.

\section{Cell culture and transwell assay}

Ly6 $\mathrm{C}^{\text {high }}$ monocytes isolated from the blood and spleen were stimulated with $30 \mathrm{ng} / \mathrm{ml}$ M-CSF (Peprotech, Rocky Hill, NJ, USA) in normal culture media (RPMI-1640, $10 \%$ FCS, NEAA, $1 \%$ PenStrep) as indicated. Ly6C ${ }^{\text {high }}$ monocytes isolated from the bone marrow were differentiated into macrophages with $30 \mathrm{ng} / \mathrm{ml} \mathrm{M-CSF}$ in normal culture media over 7 days and then stimulated with $10 \mu \mathrm{g} /$ $\mathrm{ml}$ human DiI-medium oxidized LDL (Kalen Biomedical, Montgomery Village, MD, USA) for $16 \mathrm{~h}$ in a minimal medium ( $2 \%$ FCS in RPMI without growth factor supplements). Foam cell formation was evaluated by microscopy and flow cytometry. Bone marrow monocytes, isolated with the EasySep Mouse Monocyte Isolation Kit (Stemcell Technologies, Cologne, Germany) according to the manufacturer's instructions, were allowed to migrate for $3 \mathrm{~h}$ in a transwell chamber $\left(2 \times 10^{5}\right.$ cells per well; Costar $6.5 \mathrm{~mm}$ Transwell inserts with $5 \mu \mathrm{m}$ pore membrane, Corning, Kennebunk, ME, USA) stimulated by CCL2 $20 \mathrm{ng} / \mathrm{ml}$, CCL5 $20 \mathrm{ng} / \mathrm{ml}$ (R\&D System, Minneapolis, MN, USA) or BSA $20 \mathrm{ng} / \mathrm{ml}$ as control. Cells in the lower chambers were quantified with an automated cell counter (Z2 analyzer, Beckman Coulter, Inc., Brea, CA, USA).

\section{RNA isolation and real-time PCR}

Cells were sorted into RLT/ $\beta$-mercaptoethanol for subsequent RNA isolation using the RNeasy Micro Kit (Qiagen, Valencia, CA, USA) according to the manufacturer's instructions. The Oviation PicSL WTA System V2 (NuGEN, San Carlos, CA, USA) was used for reverse transcription and cDNA amplification according to the manufacturer's instructions. Quantitative TaqMan-PCR was run on a Bio-Rad CFX96 Touch Real-Time PCR System (Bio-Rad Laboratories, Hercules, CA, USA). The specific TaqMan probes used are listed in the supplemental method section.

\section{Statistics}

Results are presented as mean \pm SEM. Differences between 2 groups were evaluated by the unpaired Student's $t$ test if they passed the Kolmogorov-Smirnov normality test or otherwise by the non-parametric Mann-Whitney test as indicated. Differences between more than 2 groups were evaluated by Kruskal-Wallis test with Dunn's multiple comparison test. $p$ values $\leq 0.05$ denote significant changes.

\section{Results}

SYK inhibition attenuates atherosclerotic plaque development in $\mathrm{Apoe}^{-/-}$mice

6-week-old $\mathrm{Apoe}^{-1-}$ mice, still devoid of atherosclerosis, consumed a high cholesterol diet (HCD) supplemented with or without $0.3 \%(\mathrm{w} / \mathrm{w})$ SYK inhibitor fostamatinib for 8 weeks. At this point, we observed de novo plaque formation in the aortic root and abdominal aorta. Histologic analysis revealed that SYK inhibition markedly reduced overall lesion size, lipid and macrophage content in the aortic root and abdominal aorta, respectively (Fig. 1a-d), despite similar plasma cholesterol levels (Supplemental Table 1). Flow cytometric analysis of aortic tissue lysates confirmed a significant reduction in Ly6 $\mathrm{C}^{\text {high }}$ monocyte and macrophage numbers (Fig. 1e, f).

\section{SYK inhibition reduces medullary}

and extramedullary myelopoiesis in atherosclerotic Apoe $^{-1-}$ mice

In accord with reduced cell counts in the aorta fostamatinib prevented the rise in circulating Ly6 $\mathrm{C}^{\text {high }}$ monocytes associated with hypercholesterolemia and atherogenesis (Fig. 2a). We queried the possible mechanisms. First, Ly6 $\mathrm{C}^{\text {high }}$ monocyte numbers failed to increase in the bone marrow and spleen after 8 weeks of HCD with fostamatinib intake (Fig. 2b) indicating hampered medullary and extramedullary myelopoiesis. Treatment with the SYK inhibitor lowered both the percentage of common myeloid progenitors (CMP) that incorporated BrdU and the frequency of their progeny, the macrophage dendritic cell progenitors (MDP), that give rise to monocytes, in the bone marrow and spleen (Fig. 2c, Supplemental Figure 1). Secondly, we found no signs of increased myelotoxicity with fostamatinib as assessed by Annexin V and PI staining (Fig. 2d). Lastly, fostamatinib-treated and control mice showed similar CCR2 expression levels on Ly6 $\mathrm{C}^{\text {high }}$ monocytes in the bone marrow and no difference in their mobilization upon intravenous CCL2 administration (Fig. 2e, f). These data indicate that fostamatinib inhibited hypercholesterolemia-associated inflammatory monocyte production.

\section{SYK inhibition blocks GM-CSF/IL-3 stimulated myelopoiesis}

Increased medullary and extramedullary monocyte production in Apoe $^{-/-}$mice results from impaired cholesterol efflux and heightened surface expression of the common 

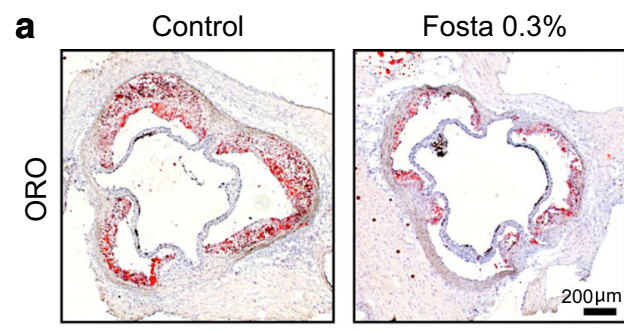

b
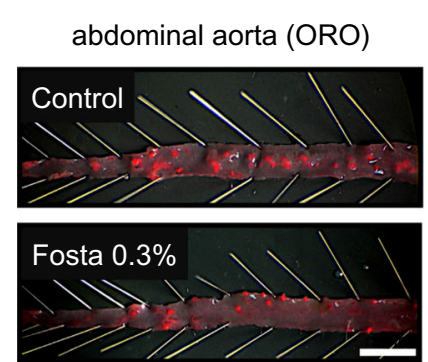

d

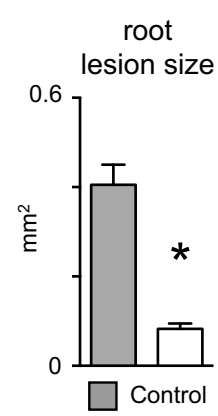

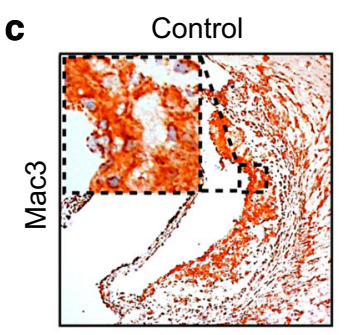
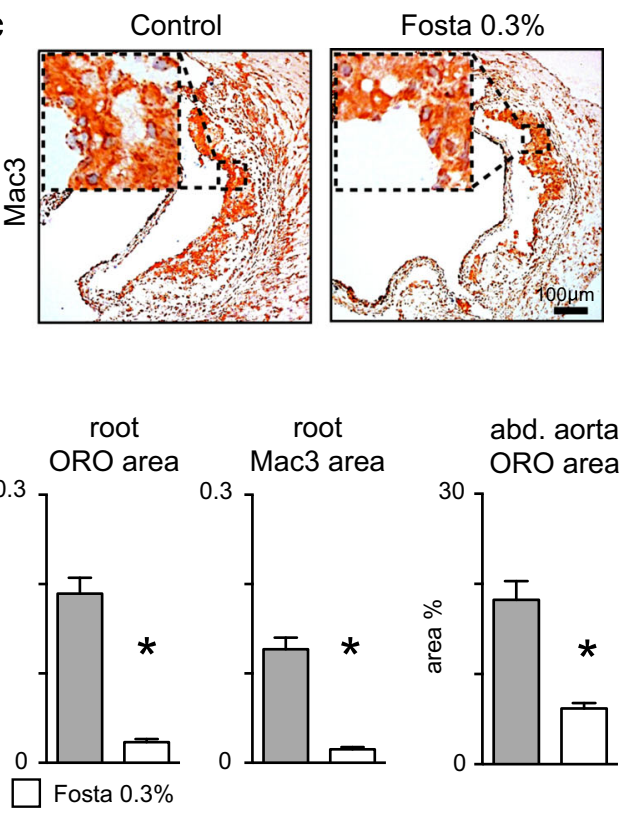

e

Control

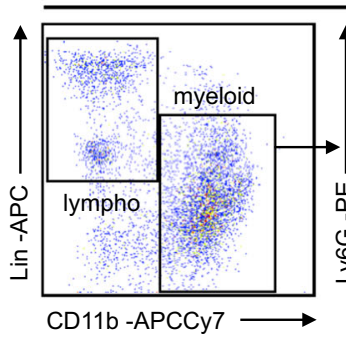

Fosta $0.3 \%$
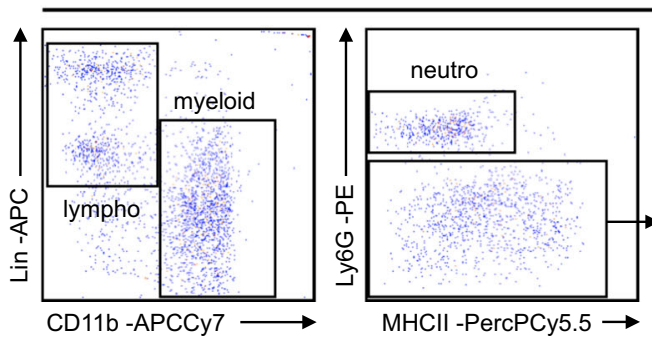

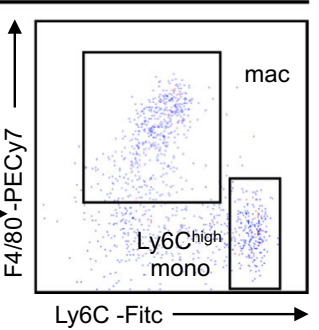

f
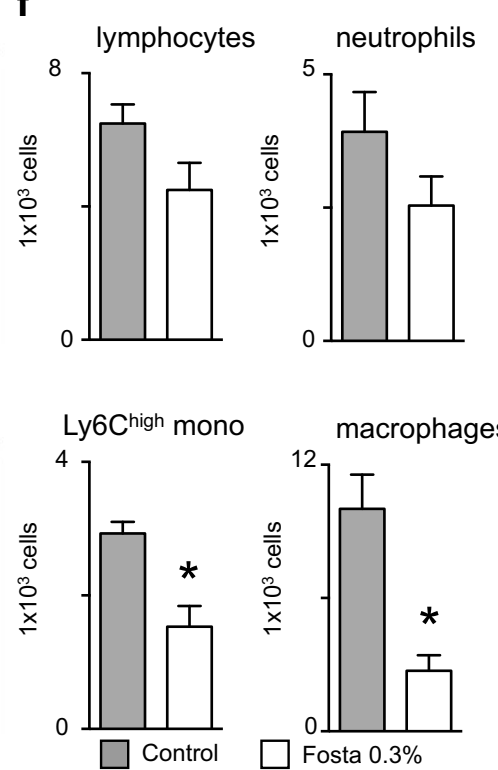

Fig. 1 Fostamatinib reduces atheroma initiation in $\mathrm{Apoe}^{-/-}$mice. a Representative Oil Red O $(O R O)$ stainings of aortic root sections and (b) abdominal aortas from control and fostamatinib $0.3 \% \mathrm{w} / \mathrm{w}$ (Fosta $0.3 \%$ ) treated Apoe ${ }^{-/-}$mice after 8 weeks of high cholesterol diet $(H C D)$. c Representative macrophage (Mac3) staining of aortic root sections with higher magnification from control and fostamatinib $0.3 \%$ treated Apoe ${ }^{-I-}$ mice after 8 weeks of HCD. d Quantification of lesion size, lipid (ORO) and macrophage (Mac3) content in aortic root lesions ( $n=14$ per group) and lesion area in abdominal aortas

beta subunit of the GM-CSF/IL-3 receptor (CBS) on progenitor cells [10, 12, 19]. GM-CSF and IL-3 stimulate myeloid progenitor cell proliferation and SYK binds to the CBS for signal transduction [18]. We observed SYK phosphorylation at $\mathrm{Tyr}^{519 / 520}$ associated with kinase activation in bone marrow CMP of Apoe ${ }^{-/-}$mice in response
( $n=8$ per group) of control (gray) and fostamatinib $0.3 \%$ (white) treated Apoe ${ }^{-I-}$ mice after 8 weeks of HCD. Results are presented as mean \pm SEM. $* p \leq 0.05, t$ test. e Analysis of aortic plaque lesions by flow cytometry and (f) quantification of lymphocytes (lympho), myeloid cells, macrophages (mac), neutrophils (neutro) and Ly6C $\mathrm{C}^{\text {high }}$ monocytes (mono) in control (gray) and fostamatinib $0.3 \%$ (white) treated Apoe $^{-1-}$ mice ( $n=6$ per group) after 8 weeks of HCD. Results are presented as mean \pm SEM. ${ }^{*} p \leq 0.05, t$ test. Lin lineage cocktail with anti-CD3, anti-CD19, anti-NK1.1

to stimulation with GM-CSF and IL-3. R406, the active metabolite of fostamatinib, limited GM-CSF/IL-3 induced SYK autophosphorylation (Fig. 3a). SYK inhibition by R406 reduced colony formation by bone marrow cells stimulated with GM-CSF and IL-3 in line with our in vivo data (Fig. 3b). 


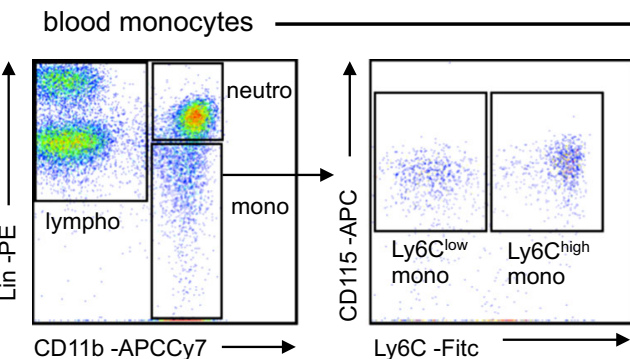

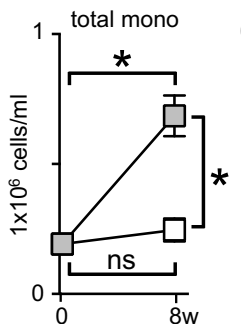

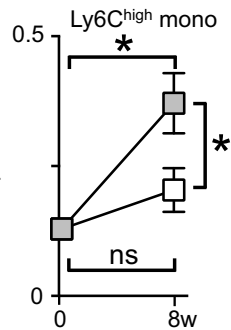

b

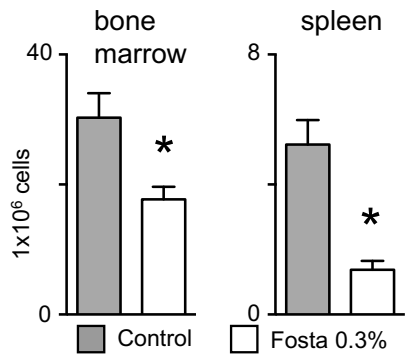

C
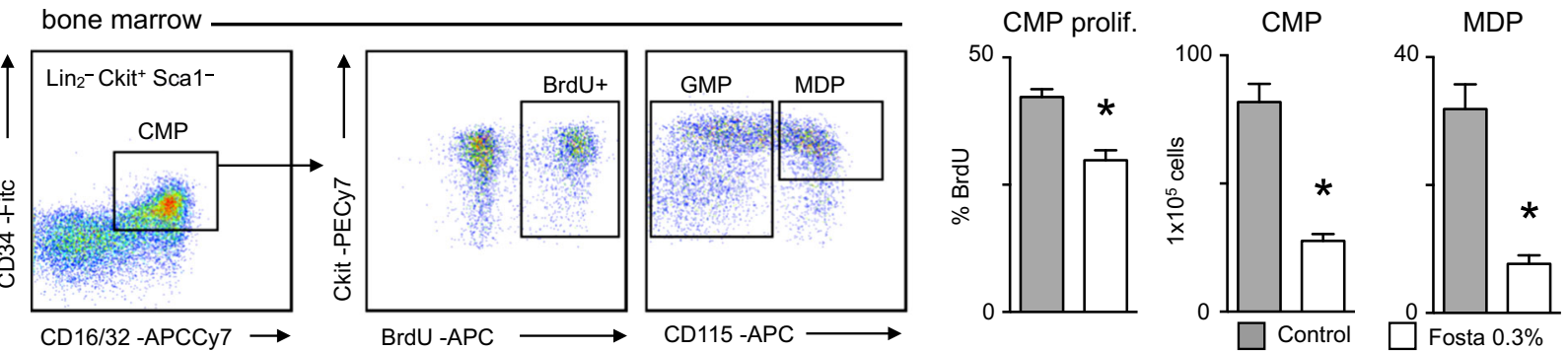

d

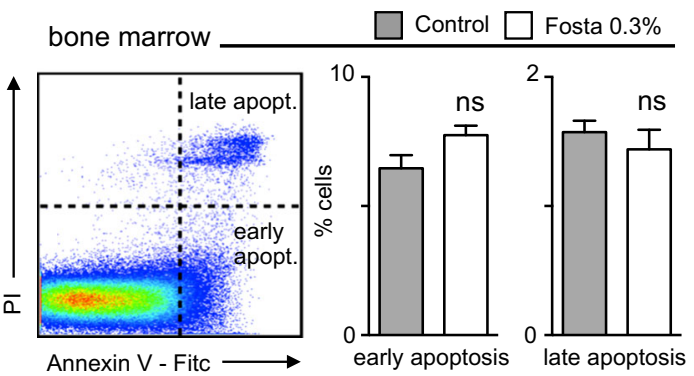

e bone marrow $\square$ Control $\square$ Fosta $0.3 \%$

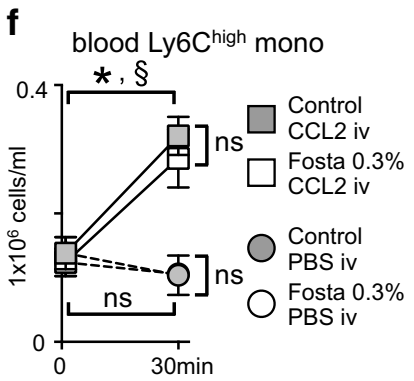

Fig. 2 Fostamatinib inhibits monocytosis in hypercholesterolemic Apoe $^{-1-}$ mice. a Identification and quantification of blood monocyte subsets by flow cytometry at baseline $(n=8)$ and after 8 weeks of HCD in control (gray) and fostamatinib $0.3 \%$ (white) treated Apoe $^{-1-}$ mice $(n=14$ per group). Results are presented as mean \pm SEM. $* p \leq 0.05$. $n s$ not significant if $p>0.05, t$ test. Lin lineage cocktail with anti-CD3, anti-CD19, anti-NK1.1, anti-Ly6G. b Quantification of Ly6 $\mathrm{C}^{\text {high }}$ monocytes in the bone marrow and spleen of control (gray) and fostamatinib $0.3 \%$ (white) treated Apoe $^{-/-}$mice after 8 weeks of $\operatorname{HCD}(n=14$ per group). Results are presented as mean \pm SEM. ${ }^{*} p \leq 0.05, t$ test. c Identification and quantification of common myeloid progenitor $(C M P)$ cell number and rate of proliferation as determined by bromodeoxyuridine $(B r d U)$ incorporation. Identification and quantification of granulocyte macrophage $(G M P)$ and macrophage dendritic cell progenitors $(M D P)$ in the bone marrow of control (gray) and fostamatinib $0.3 \%$ (white) treated Apoe $^{-1-}$ mice ( $n=10$ per group) after 8 weeks of HCD. Results are presented as mean \pm SEM. ${ }^{*} p \leq 0.05, t$ test. Lin $_{2}$ lineage cocktail with anti-CD3, anti-CD90.2, anti-CD19, anti-NK1.1, anti-

\section{SYK inhibition attenuates monocyte infiltration and differentiation but not macrophage egress from atherosclerotic lesions}

We next studied possible local effects of fostamatinib on lesional macrophage accumulation apart from monocyte production and mobilization. Consumption of HCD supplemented with $0.3 \%$ fostamatinib for 4 days did not
CD49b, anti-Gr-1, anti-CD11b, anti-CD11c, anti-IL7Ra. d Identification and quantification of early and late bone marrow cell apoptosis of control (gray) and fostamatinib $0.3 \%$ (white) treated Apoe ${ }^{-/-}$mice ( $n=10$ per group) after 8 weeks of HCD by Annexin V and PI staining. Results are presented as mean \pm SEM. $n s$ not significant if $p>0.05, t$ test. e Quantification of CCR 2 mean fluorescence intensity on Ly6 $\mathrm{C}^{\text {high }}$ bone marrow monocytes of control (gray) and fostamatinib $0.3 \%$ (white) treated Apoe ${ }^{-1-}$ mice $(n=10$ per group) after 8 weeks of HCD. Results are presented as mean \pm SEM. $n s$ not significant if $p>0.05, t$ test. f Apoe ${ }^{-/-}$mice consumed a HCD with or without fostamatinib $0.3 \%$ for 4 days, when peripheral monocyte numbers were still unaffected. Ly6 $\mathrm{C}^{\text {high }}$ monocytes of control (gray) and fostamatinib $0.3 \%$ (white) treated Apoe ${ }^{-1-}$ mice were counted in the blood before and $30 \mathrm{~min}$ after intravenous CCL2 ( $n=6$ per group; squares) or PBS ( $n=4$ per group; circles) administration. Results are presented as mean \pm SEM. $* p \leq 0.05$ within the control group and ${ }^{\S} p \leq 0.05$ within the fostamatinib group, Kruskal-Wallis test. $n s$ not significant if $p>0.05$

lower the number of leukocytes in the circulation but reduced their rolling along and adhesion to the intimal surface as assessed by intravital microscopy of TNF $\alpha$ stimulated mesenteric veins (Fig. 4a). SYK inhibition, however, did not interfere with CCL2 and CCL5 directed monocyte migration in a transwell chamber (Fig. 4b). The reduced number of lesional macrophages observed in fostamatinib-treated $\mathrm{Apoe}^{-/-}$mice (Fig. 1e, f) may not only 
a bone marrow
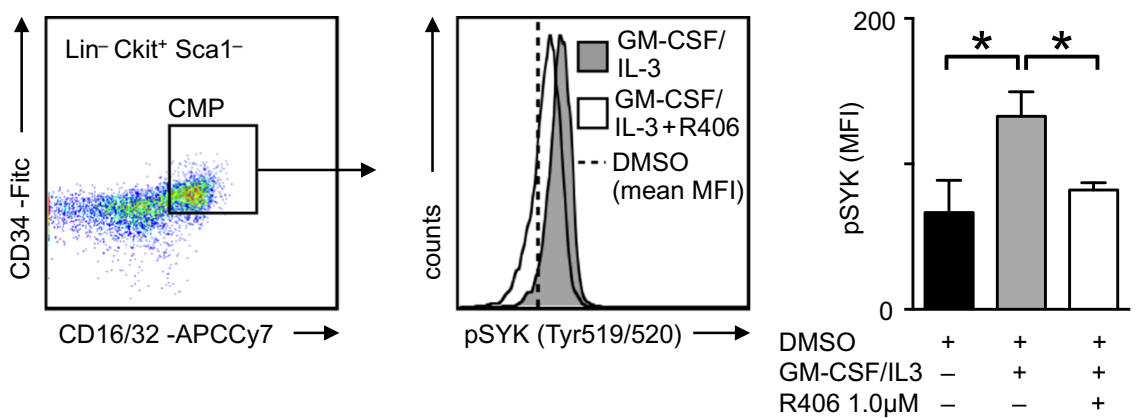

b
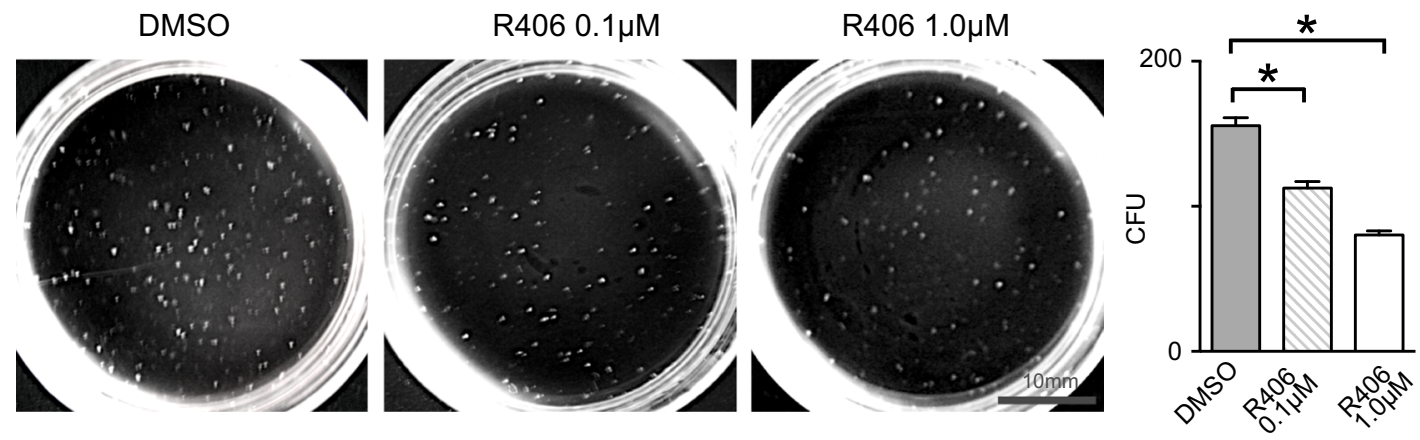

Fig. 3 The monocytopoietic growth factors GM-CSF and IL3 activate SYK. a Quantification of SYK phosphorylation (Tyr519/ 520) in bone marrow common myeloid progenitor cells isolated from Apoe $^{-l-}$ mice and stimulated with GM-CSF and IL3 for $10 \mathrm{~min}$ in the presence (white) or absence (gray) of SYK inhibitor R406 as determined by flow cytometry. Unstimulated DMSO controls are depicted as a dashed line and black bar, respectively $(n=3$ duplicates per group). Results are presented as mean \pm SEM.

result from reduced monocyte availability inside the lesion but also from impaired monocyte to macrophage differentiation or increased macrophage egress. Monocyte to macrophage differentiation depends on M-CSF. Stimulation of mature Ly6 $\mathrm{C}^{\text {high }}$ monocytes isolated from the blood and spleens of Apoe ${ }^{-l-}$ mice with M-CSF induced SYK activation and phosphorylation at $\mathrm{Tyr}^{519 / 520}$ that was abrogated by R406 (Fig. 4c). Consequently, overnight culture of sorted Ly6C $\mathrm{C}^{\text {high }}$ monocytes with M-CSF and SYK inhibitor R406 blocked their differentiation into live F4/80 high macrophage-like cells (Fig. 4d). To evaluate macrophage egress from atherosclerotic lesions we injected fluorescent beads, as previously described [11], into young Apoe $^{-1-}$ mice that had just been placed on HCD 4 days previously (Fig. 4e). Within 6 days myeloid cells had cleared the blood from circulating beads and infiltrated the nascent plaques (Fig. 4f). Fostamatinib treatment was started at this time point in one group while the other group continued on HCD for another 8 weeks, thus avoiding any influence of the drug on the initial cell mediated accumulation of beads in lesions (Fig. 4e). The beads are nondegradable and can only leave the lesions within
$* p \leq 0.05$, Kruskal-Wallis test. Lin lineage cocktail with anti-CD3, anti-CD90.2, anti-CD19, anti-NK1.1, anti-CD49b, anti-Gr-1, antiCD11b, anti-CD11c, anti-IL7Ra. b Colony forming unit (CFU) assay of bone marrow cells isolated from Apoe ${ }^{-1-}$ mice stimulated with GM-CSF and IL3 in the presence or absence (gray) of SYK inhibitor $\mathrm{R} 406(0.1 \mu \mathrm{M}$ in shaded, $1.0 \mu \mathrm{M}$ in white). Results are presented as mean \pm SEM for $n=4$ duplicates per group. ${ }^{*} p \leq 0.05$, KruskalWallis test

emigrating phagocytes [11]. Therefore, the number of beads remaining in the lesion inversely correlates with macrophage egress. 8 weeks of fostamatinib treatment did not change the number of beads in lesions but still reduced the lesional macrophage content (Fig. 4g, Supplemental Figure 2). We concluded that SYK inhibition mainly limited the number of infiltrating monocytes and their differentiation but not macrophage emigration from atherosclerotic lesions.

\section{SYK inhibition exerts subtle effects on macrophage foam cell formation and inflammation}

Macrophages accumulate lipids in atherosclerotic lesions and develop into foam cells. We tested if SYK inhibition affected foam cell formation. To this end, we generated Ly6 $\mathrm{C}^{\text {high }}$ monocyte derived macrophages that were incubated with DiI-labeled oxidized low density lipoprotein (DiI-oxLDL) overnight in the presence or absence of SYK inhibitor R406. The number of macrophages in culture remained unchanged, and all viable macrophages ingested oxLDL, though SYK inhibition reduced the mean 
a

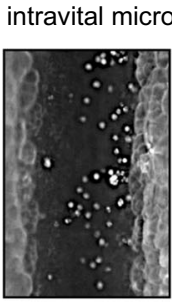

Control

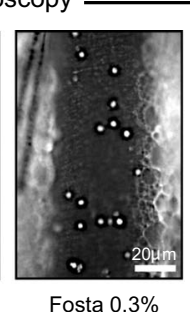

Fosta $0.3 \%$

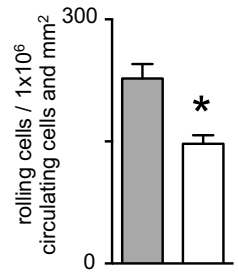

$\square$ Control

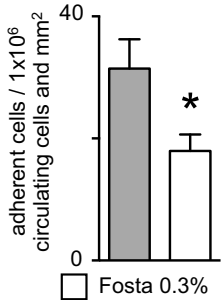

d

b monocyte chemotaxis

C
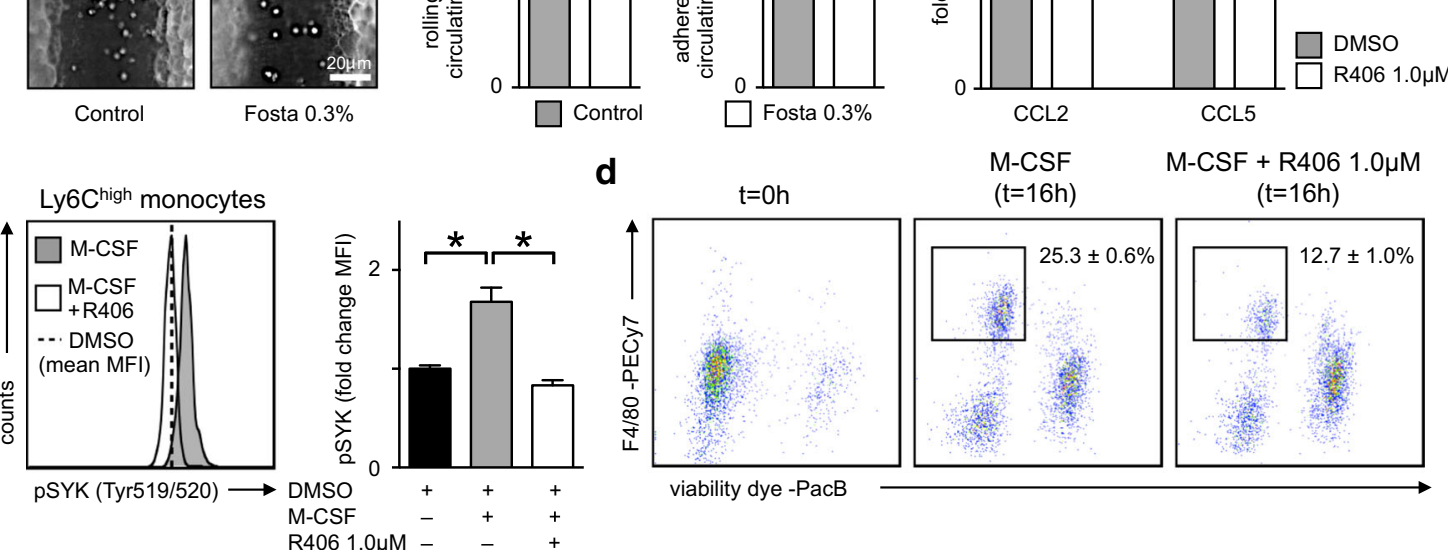

M-CSF

CCL5 $(\mathrm{t}=16 \mathrm{~h})$

$\mathrm{M}-\mathrm{CSF}+\mathrm{R} 4061.0 \mu \mathrm{M}$ $(\mathrm{t}=16 \mathrm{~h})$
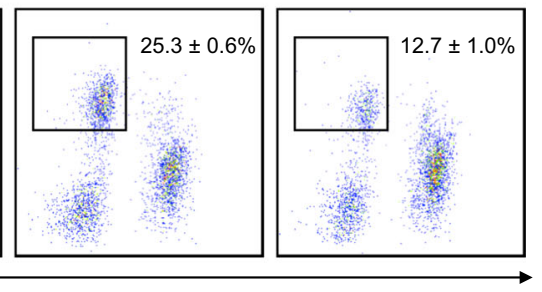

g
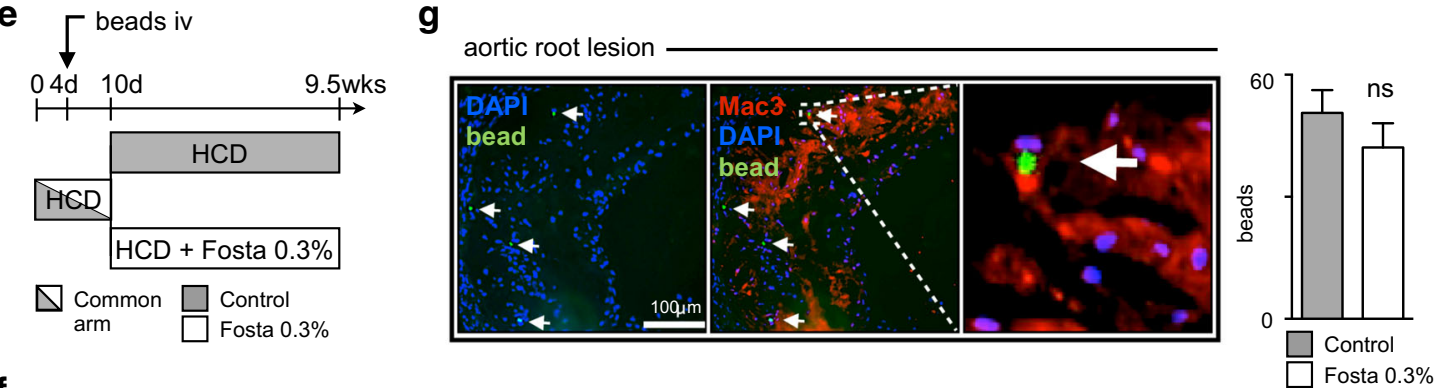

f

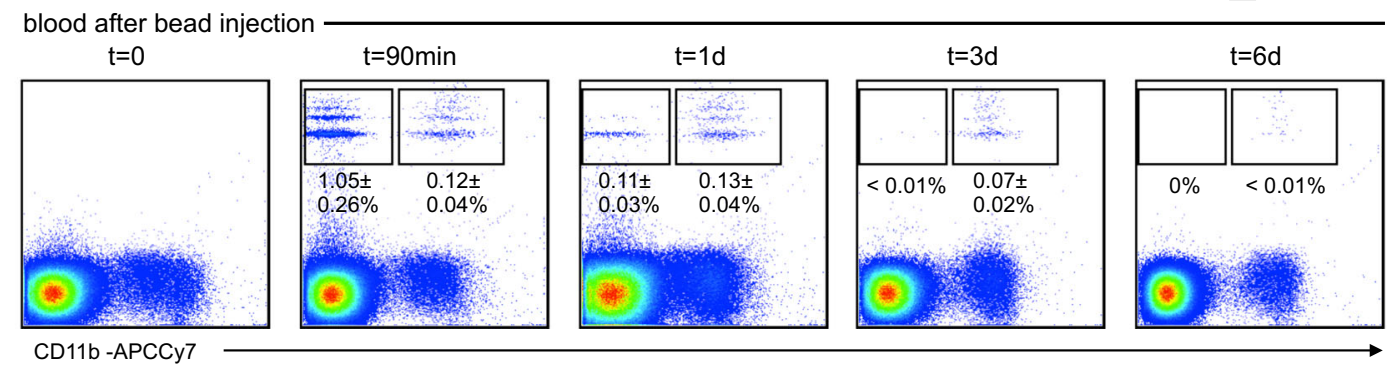

Fig. 4 Fostamatinib inhibits de novo macrophage accumulation but not egress from atherosclerotic lesions. a Quantification of rolling and adherent leukocytes by intravital microscopy of mesenteric veins in control (gray) and fostamatinib $0.3 \%$ (white) treated Apoe ${ }^{-1-}$ mice (4 days of HCD) $3 \mathrm{~h}$ after intraperitoneal TNF $\alpha$ administration. Results are normalized to individual peripheral cell counts and the vessel segment areas analyzed, and are presented as mean \pm SEM, $n>50$ vessel segments per group. ${ }^{*} p \leq 0.05, t$ test. b Quantification of CCL2 and CCL5 stimulated monocyte migration over BSA in a transwell chamber. Results are presented as mean \pm SEM, $n=5$ per group. ${ }^{*} p \leq 0.05, t$ test. c Quantification of SYK phosphorylation (Tyr519/520) in Ly6C $\mathrm{C}^{\text {high }}$ blood monocytes of $\mathrm{Apoe}^{-/-}$mice stimulated with M-CSF for $10 \mathrm{~min}$ in the presence (white) or absence (gray) of SYK inhibitor R406 as determined by flow cytometry. Unstimulated DMSO controls are depicted as a dashed line and black bar, respectively ( $n=3$ duplicates per group). Results are presented as mean \pm SEM. $* p \leq 0.05$, Kruskal-Wallis test. d Ly6C ${ }^{\text {high }}$ monocytes isolated from the blood and spleen $(t=0 \mathrm{~h})$ were stimulated with M-CSF in the presence or absence of SYK inhibitor R406 for $16 \mathrm{~h}$. The fraction of viable cells that upregulated macrophage marker F4/80 was quantified by flow cytometry. Results are presented as mean \pm SEM, $n=5$ per group, $p<0.01, t$ test. e Treatment scheme of Apoe ${ }^{-l-}$ mice with HCD for 10 days (bicolored, common) before randomization to fostamatinib $0.3 \%$ (white) or continued HCD (gray) for another 8 weeks. Fluorescent beads were injected intravenously on day 4. f Quantification of fluorescent beads freely circulating or ingested by $\mathrm{CD}_{11} \mathrm{~b}^{+}$myeloid cells in the blood at indicated time points after intravenous administration. Results are presented as mean \pm SEM, $n=3$. g Identification and quantification of fluorescent beads in aortic root lesions after 9.5 weeks of HCD in control (gray) and fostamatinib $0.3 \%$ (white) treated Apoe ${ }^{-/-}$mice ( $n=8$ per group). Representative immunohistologic staining for macrophages (Mac3), nuclei (DAPI) and fluorescent beads (green) on the left and quantification of beads within lesions of 8 adjacent aortic root sections per mouse. Results are presented as mean \pm SEM. $* p \leq 0.05, t$ test 
fluorescence intensity of DiI by one-third (Fig. 5a, b), similar to what had previously been observed with other lipoprotein modifications [2]. We next asked whether fostamatinib altered the inflammatory phenotype of monocytes and macrophages in the context of atherosclerosis. Macrophages and Ly6 $\mathrm{C}^{\text {high }}$ monocytes were sorted from atherosclerotic aortas alongside $\mathrm{Ly} 6 \mathrm{C}^{\text {high }}$ monocytes from the blood after 8 weeks of HCD with our without fostamatinib. RNA was isolated for gene expression analysis. Overall, fostamatinib exerted rather subtle and fluctuating changes of the inflammatory profile in all cell types tested, except for significantly reduced (pro-)IL-1 $\beta$ expression by macrophages (Fig. 5c).

\section{SYK inhibition fails to prevent plaque progression}

Next, we asked whether SYK inhibition by fostamatinib could modulate progression of established plaques. Apoe $^{-/-}$mice consumed a HCD for 8 weeks to allow plaque formation throughout the aorta (common group). Then mice were randomized to either HCD alone or HCD supplemented with $0.3 \%(\mathrm{w} / \mathrm{w})$ fostamatinib for another 12 weeks (Fig. 6a). Again, treatment with fostamatinib abrogated the continuous rise of Ly6 $\mathrm{C}^{\text {high }}$ monocytes in the blood, bone marrow and spleen (Fig. 6b, c). In contrast,

a

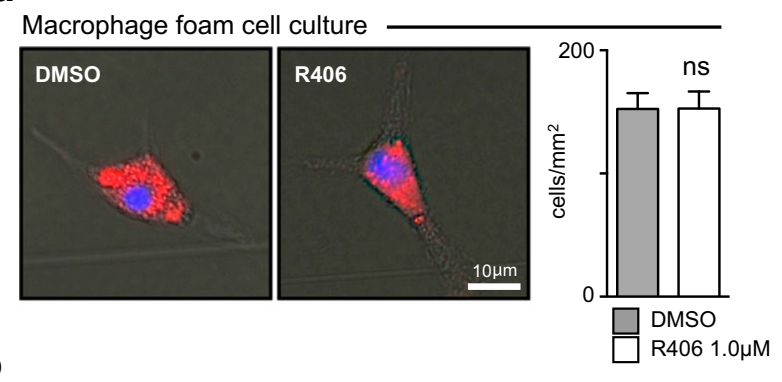

b

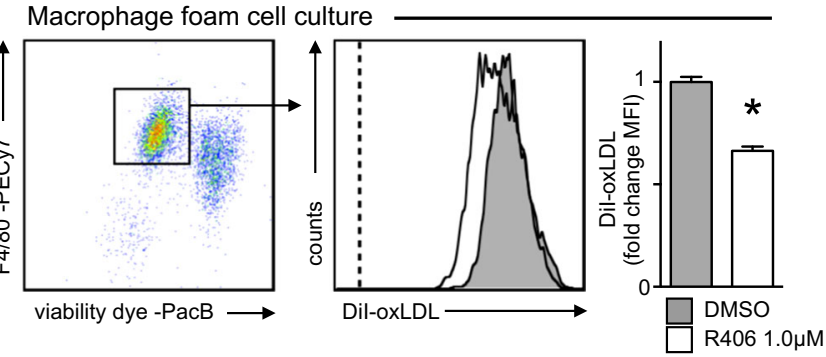

Fig. 5 Fostamatinib exerts subtle effects on macrophage foam cell formation and inflammation. a, b Ly6 $\mathrm{C}^{\text {high }}$ monocytes were stimulated with M-CSF to generate macrophages in culture before incubation with DiI-oxLDL under starving conditions in the presence (white) or absence (gray) of SYK inhibitor R406. a Identification and quantification of macrophage foam cells by immunofluorescence microscopy. Representative images are shown on the left. Results are presented on the right as mean \pm SEM. $* p \leq 0.05, t$ test. b Quantification of DiI-oxLDL accumulation by flow cytometry. Results are fostamatinib intake did not alter lesion progression in the root and abdominal aorta. Lesional lipid and macrophage contents did not differ between groups (Fig. 6d-g). To address the dissociation between circulating monocytes and lesional macrophage accumulation we quantified macrophage proliferation and cell death in aortic root sections. Fostamatinib treatment did not change the percentage of proliferating $\mathrm{Ki}^{+} 7^{+}$and apoptotic $\mathrm{TUNEL}^{+}$ macrophages in the plaque (Fig. 6h, i, Supplemental Figure 3).

\section{Discussion}

We show here that SYK inhibition by fostamatinib differentially interfered with the turnover and accumulation of monocytes and macrophages in atherosclerotic lesions on multiple levels. Fostamatinib impaired hypercholesterolemia-associated monocytosis stimulated by GM-CSF and IL-3 in $\mathrm{Apoe}^{-1-}$ mice. In concert with reduced integrin-mediated cell adhesion $[6,9,16]$ and hampered M-CSF-dependent macrophage differentiation, fostamatinib potently inhibited the early accumulation of lesional macrophages and the development of new atherosclerotic lesions by more than $70 \%$. Chemoattraction mediating
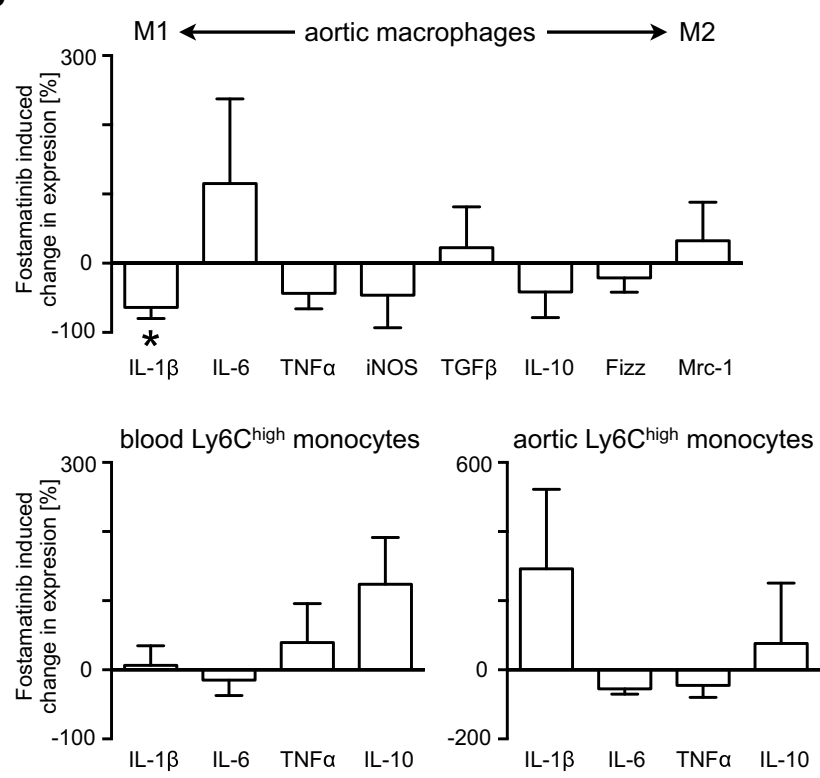

presented as mean \pm SEM. $* p \leq 0.05$, Mann-Whitney test. c Gene expression profiling of macrophages and Ly6 $\mathrm{C}^{\text {high }}$ monocytes isolated from atherosclerotic aortas and blood, respectively, after 8 weeks of HCD in control and fostamatinib $0.3 \%$ treated Apoe ${ }^{-1-}$ mice $(n=6$ per group). Results are presented as mean \pm SEM percent change of marker expression in fostamatinib-treated mice compared to controls. $* p \leq 0.05, t$ test. Other changes are statistically not significant 
a

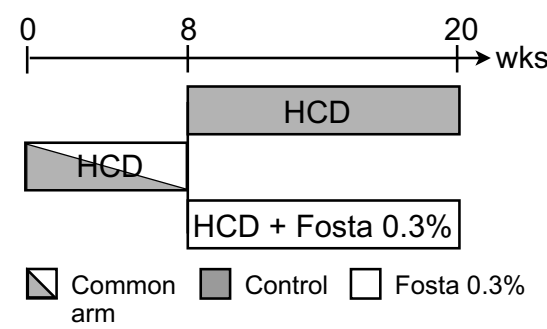

b

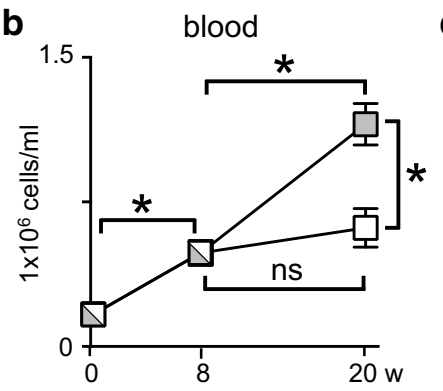

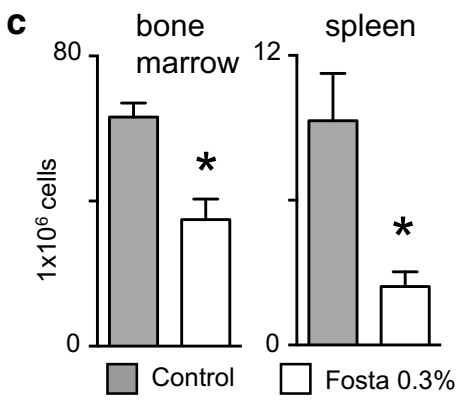

d
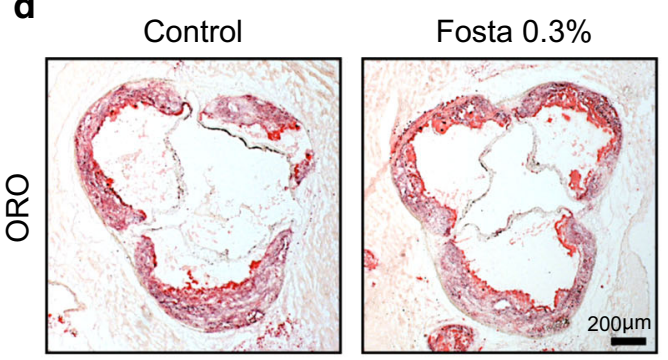

f
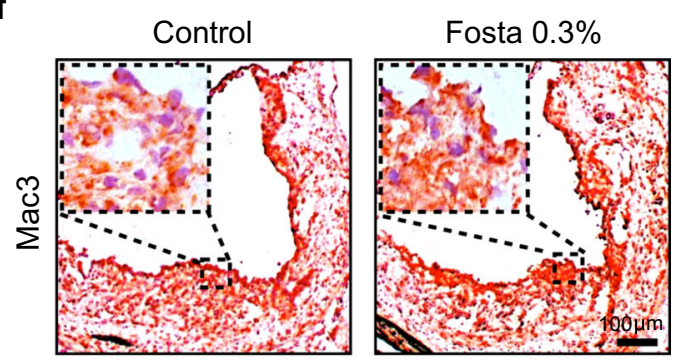

e
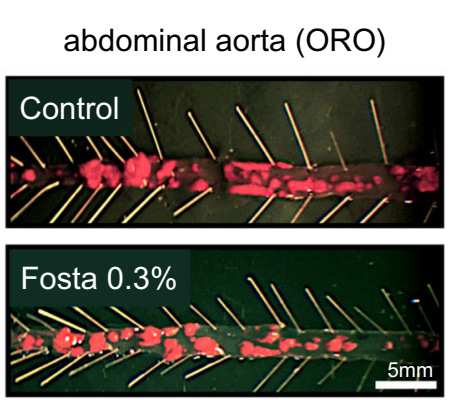

g

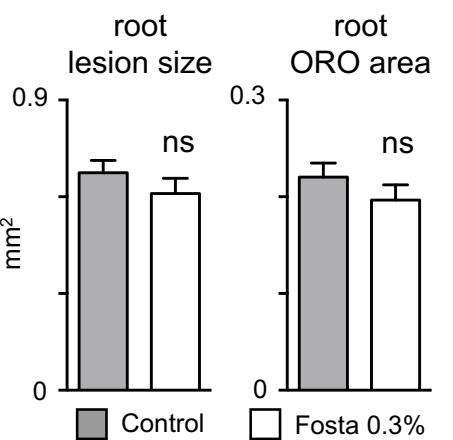

h

aortic root lesion
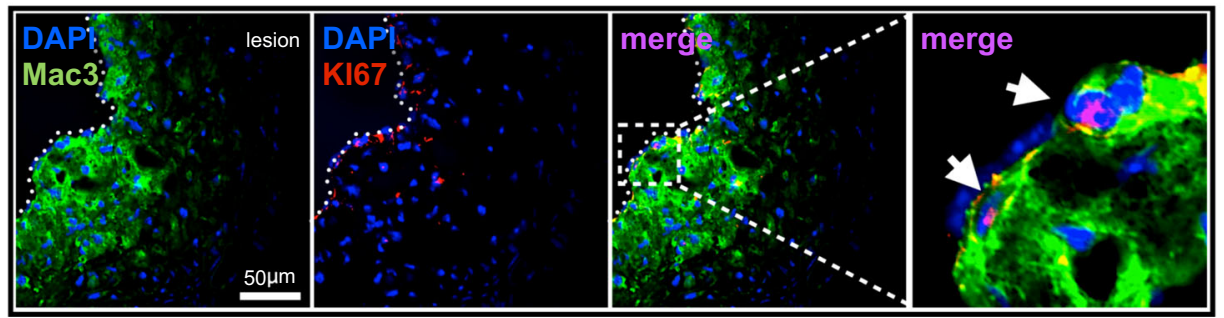

root

abd. aorta

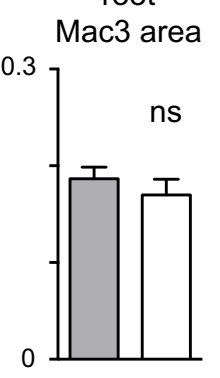

ORO area

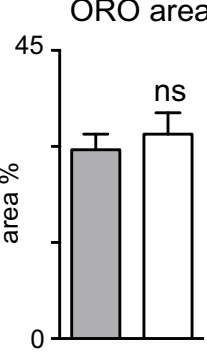

i $\mathrm{Ki}^{6} 7^{+} \mathrm{Mac3}^{+}$

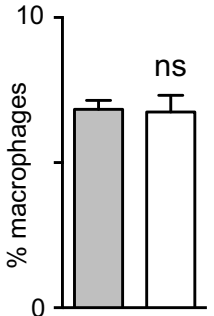

Fig. 6 Continued lesion progression despite fostamatinib treatment with reduced monocytosis in established atherosclerosis. a Treatment scheme of Apoe ${ }^{-1-}$ mice with Fosta $0.3 \%$ (white) or continued HCD (gray) over 12 weeks after a prior 8 week HCD feeding period to establish plaque development (bicolored, common). b Quantification of blood Ly6C ${ }^{\text {high }}$ monocytes by flow cytometry at baseline, after 8 weeks of HCD (bicolored, common) and after an additional 12 weeks of HCD with (white) or without (gray) Fosta $0.3 \%$ supplementation ( $n=18$ per group). Results are presented as mean \pm SEM. ${ }^{*} p \leq 0.05$. $n s$ not significant if $p>0.05$. c Quantification of Ly6 $\mathrm{C}^{\text {high }}$ monocytes in the bone marrow and spleen of control (gray) and Fosta $0.3 \%$ (white) treated Apoe ${ }^{-1-}$ mice after $8+12$ weeks of HCD ( $n=12$ per group). Results are presented as mean \pm SEM. $* p \leq 0.05$. d Representative Oil Red O $(O R O)$ stainings of aortic root sections and (e) abdominal aortas from control and Fosta $0.3 \%$ treated Apoe ${ }^{-1-}$ mice after $8+12$ weeks of HCD. f Representative macrophage (Mac3) staining of aortic root sections with higher magnification from control and fostamatinib $0.3 \%$ treated Apoe ${ }^{-1-}$ mice after $8+12$ weeks of HCD. g Quantification of lesion size, lipid (ORO) and macrophage (Mac3) content in aortic root lesions and of lesion area in abdominal aortas $(n=18$ per group) of control (gray) and fostamatinib $0.3 \%$ (white) treated Apoe $^{-l-}$ mice after $8+12$ weeks of HCD. Results are presented as mean \pm SEM. $n s$ not significant if $p>0.05$. h Representative immunohistologic staining for macrophages (Mac3), nuclei (DAPI) and proliferation marker Ki67 of aortic root sections from control and Fosta $0.3 \%$ treated Apoe $^{-/-}$mice after $8+12$ weeks of HCD. i Percentage of KI67 + proliferating lesional macrophages $(n \geq 50$ visual fields per group). Results are presented as mean \pm SEM. $n s$ not significant if $p>0.05$ 


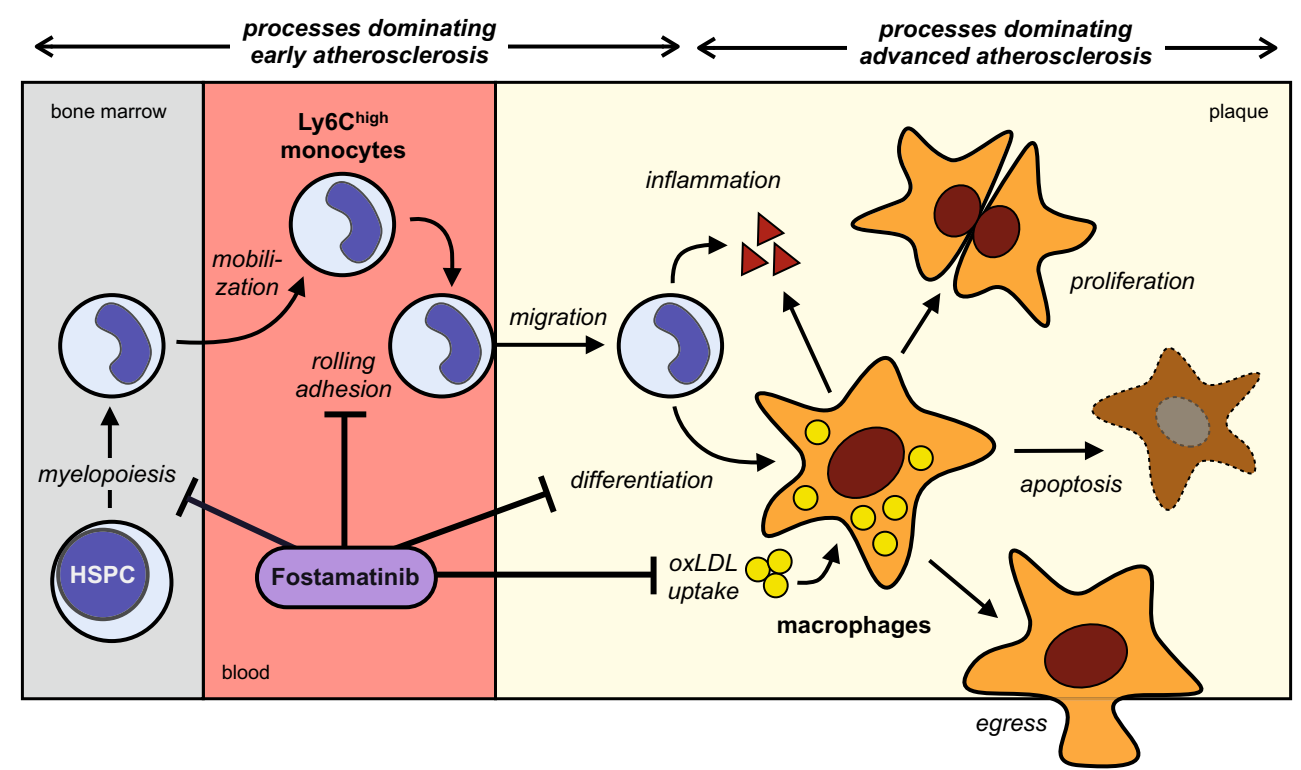

Fig. 7 A model of selective fostamatinib effects on monocyte/macrophage dynamics and function in atherosclerosis

monocyte/macrophage mobilization and migration involves G-protein-coupled receptor signaling [8, 20] independent of SYK and, therefore, may have remained unaffected. Our findings agree with the concept that macrophages in early atherosclerosis directly derive from infiltrating Ly6C ${ }^{\text {high }}$ monocytes [7]. In established lesions, however, local foam cell proliferation rather than monocyte recruitment and differentiation dominates macrophage turnover [13]. SYK inhibition by fostamatinib, when started after lesions had already formed, still reduced Ly6 $\mathrm{C}^{\text {high }}$ monocytosis by about $50 \%$. Fewer monocytes, however, did not translate into reduced macrophage accumulation in advanced lesions. In fact, lesions continued to grow throughout the aorta by more than $50 \%$ even with fostamatinib treatment. Half the amount of circulating Ly6C $\mathrm{C}^{\text {high }}$ monocytes might still suffice to sustain the macrophage pool in atherosclerotic lesions of fostamatinib-treated mice. We propose, however, that the failure to influence intraplaque macrophage proliferation, or cell death or egress rendered fostamatinib treatment ineffective in established disease (Fig. 7). While GM-CSF/IL-3 stimulate myeloid progenitor cell proliferation via SYK, macrophage proliferation in more advanced lesions does not depend on GMCSF/IL-3 signaling [13]. Rheumatoid arthritis and atherosclerosis share important common pathogenic mechanisms, and GM-CSF drives monocytosis in experimental rheumatoid arthritis [3]. When rheumatoid patients are treated with fostamatinib blood monocyte levels drop by $36 \%$ [5].

Our study indicates that the treatment of atherosclerosis may require disease stage specific approaches. Interventions directed against monocyte production and recruitment may limit early lesion formation or acute aggravation. In advanced lesions, however, when local proliferation dominates macrophage accumulation, targeting cell division itself or the respective stimulators may prove more effective. This concept, if applicable to humans, has major clinical implications as patients with atherosclerosis usually become symptomatic only after plaques have already formed.

Acknowledgments We thank Jennifer Buchholz and Diana Selig for editorial assistance. We thank Esteban Masuda, Rigel Pharmaceuticals Inc. for his critical review.

\section{Compliance with ethical standards}

\section{Conflict of interest None.}

Funding This work was supported by internal funds of the Department of Cardiology and Angiology I, University Heart Center Freiburg, and Astra Zeneca to A.Z., the German Research Foundation to I.H. (HI 1573/2-1) and the Excellence Initiative of the German Research Foundation (GSC-4, Spemann Graduate School) to K.D.B.

Open Access This article is distributed under the terms of the Creative Commons Attribution 4.0 International License (http://crea tivecommons.org/licenses/by/4.0/), which permits unrestricted use, distribution, and reproduction in any medium, provided you give appropriate credit to the original author(s) and the source, provide a link to the Creative Commons license, and indicate if changes were made.

\section{References}

1. Cochain C, Zernecke A (2015) Macrophages and immune cells in atherosclerosis: recent advances and novel concepts. Basic Res Cardiol 110:34. doi:10.1007/s00395-015-0491-8 
2. Choi SH, Harkewicz R, Lee JH, Boullier A, Almazan F, Li AC, Witztum JL, Bae YS, Miller YI (2009) Lipoprotein accumulation in macrophages via toll-like receptor-4-dependent fluid phase uptake. Circ Res 104:1355-1363. doi:10.1161/CIRCRESAHA. 108.192880

3. Cook AD, Turner AL, Braine EL, Pobjoy J, Lenzo JC, Hamilton JA (2011) Regulation of systemic and local myeloid cell subpopulations by bone marrow cell-derived granulocyte-macrophage colony-stimulating factor in experimental inflammatory arthritis. Arthritis Rheum 63:2340-2351. doi:10.1002/art.30354

4. Del Rincon I, Polak JF, O'Leary DH, Battafarano DF, Erikson JM, Restrepo JF, Molina E, Escalante A (2015) Systemic inflammation and cardiovascular risk factors predict rapid progression of atherosclerosis in rheumatoid arthritis. Ann Rheum Dis 74:1118-1123. doi:10.1136/annrheumdis-2013-205058

5. Genovese MC, van der Heijde DM, Keystone EC, Spindler AJ, Benhamou C, Kavanaugh A, Fudman E, Lampl K, O'Brien C, Duffield EL, Poiley J, Weinblatt ME (2014) A phase III, multicenter, randomized, double-blind, placebo-controlled, parallelgroup study of 2 dosing regimens of fostamatinib in patients with rheumatoid arthritis with an inadequate response to a tumor necrosis factor-alpha antagonist. J Rheumatol 41:2120-2128. doi:10.3899/jrheum. 140238

6. Hilgendorf I, Eisele S, Remer I, Schmitz J, Zeschky K, Colberg C, Stachon P, Wolf D, Willecke F, Buchner M, Zirlik K, OrtizRodriguez A, Lozhkin A, Hoppe N, von zur Muhlen C, zur Hausen A, Bode C, Zirlik A (2011) The oral spleen tyrosine kinase inhibitor fostamatinib attenuates inflammation and atherogenesis in low-density lipoprotein receptor-deficient mice. Arterioscler Thromb Vasc Biol 31:1991-1999. doi:10.1161/ ATVBAHA.111.230847

7. Hilgendorf I, Swirski FK, Robbins CS (2015) Monocyte fate in atherosclerosis. Arterioscler Thromb Vasc Biol 35:272-279. doi:10.1161/ATVBAHA.114.303565

8. Keul P, Lucke S, von Wnuck Lipinski K, Bode C, Graler M, Heusch G, Levkau B (2011) Sphingosine-1-phosphate receptor 3 promotes recruitment of monocyte/macrophages in inflammation and atherosclerosis. Circ Res 108:314-323. doi:10.1161/CIR CRESAHA.110.235028

9. Mocsai A, Abram CL, Jakus Z, Hu Y, Lanier LL, Lowell CA (2006) Integrin signaling in neutrophils and macrophages uses adaptors containing immunoreceptor tyrosine-based activation motifs. Nat Immunol 7:1326-1333. doi:10.1038/ni1407

10. Murphy AJ, Akhtari M, Tolani S, Pagler T, Bijl N, Kuo CL, Wang M, Sanson M, Abramowicz S, Welch C, Bochem AE, Kuivenhoven JA, Yvan-Charvet L, Tall AR (2011) ApoE regulates hematopoietic stem cell proliferation, monocytosis, and monocyte accumulation in atherosclerotic lesions in mice. J Clin Invest. 121:4138-4149. doi:10.1172/JCI57559

11. Potteaux S, Gautier EL, Hutchison SB, van Rooijen N, Rader DJ, Thomas MJ, Sorci-Thomas MG, Randolph GJ (2011) Suppressed monocyte recruitment drives macrophage removal from atherosclerotic plaques of Apoe ${ }^{-/-}$mice during disease regression. J Clin Invest. 121:2025-2036. doi:10.1172/JCI43802

12. Robbins CS, Chudnovskiy A, Rauch PJ, Figueiredo JL, Iwamoto Y, Gorbatov R, Etzrodt M, Weber GF, Ueno T, van Rooijen N, Mulligan-Kehoe MJ, Libby P, Nahrendorf M, Pittet MJ, Weissleder R, Swirski FK (2012) Extramedullary hematopoiesis generates Ly-6C(high) monocytes that infiltrate atherosclerotic lesions. Circulation 125:364-374. doi:10.1161/CIRCULATIO NAHA. 111.061986

13. Robbins CS, Hilgendorf I, Weber GF, Theurl I, Iwamoto Y, Figueiredo JL, Gorbatov R, Sukhova GK, Gerhardt LM, Smyth D, Zavitz CC, Shikatani EA, Parsons M, van Rooijen N, Lin HY, Husain M, Libby P, Nahrendorf M, Weissleder R, Swirski FK (2013) Local proliferation dominates lesional macrophage accumulation in atherosclerosis. Nat Med 19:1166-1172. doi:10.1038/ nm.3258

14. Solomon DH, Karlson EW, Rimm EB, Cannuscio CC, Mandl LA, Manson JE, Stampfer MJ, Curhan GC (2003) Cardiovascular morbidity and mortality in women diagnosed with rheumatoid arthritis. Circulation 107:1303-1307. doi:10.1161/01.CIR. 0000054612.26458.B2

15. Swirski FK, Libby P, Aikawa E, Alcaide P, Luscinskas FW, Weissleder R, Pittet MJ (2007) Ly-6Chi monocytes dominate hypercholesterolemia-associated monocytosis and give rise to macrophages in atheromata. J Clin Invest. 117:195-205. doi:10. 1172/JCI29950

16. Vines CM, Potter JW, Xu Y, Geahlen RL, Costello PS, Tybulewicz VL, Lowell CA, Chang PW, Gresham HD, Willman CL (2001) Inhibition of beta 2 integrin receptor and Syk kinase signaling in monocytes by the Src family kinase Fgr. Immunity 15:507-519. doi:10.1016/S1074-7613(01)002221-7

17. Weinblatt ME, Genovese MC, Ho M, Hollis S, RosiakJedrychowicz K, Kavanaugh A, Millson DS, Leon G, Wang M, van der Heijde D (2014) Effects of fostamatinib, an oral spleen tyrosine kinase inhibitor, in rheumatoid arthritis patients with an inadequate response to methotrexate: results from a phase III, multicenter, randomized, double-blind, placebo-controlled, parallel-group study. Arthritis Rheumatol 66:3255-3264. doi:10. 1002/art.38851

18. Yousefi S, Hoessli DC, Blaser K, Mills GB, Simon HU (1996) Requirement of Lyn and Syk tyrosine kinases for the prevention of apoptosis by cytokines in human eosinophils. J Exp Med 183:1407-1414. doi:10.1084/jem.183.4.1407

19. Yvan-Charvet L, Pagler T, Gautier EL, Avagyan S, Siry RL, Han S, Welch CL, Wang N, Randolph GJ, Snoeck HW, Tall AR (2010) ATP-binding cassette transporters and HDL suppress hematopoietic stem cell proliferation. Science 328:1689-1693. doi:10.1126/science. 1189731

20. Zernecke A, Weber C (2014) Chemokines in atherosclerosis: proceedings resumed. Arterioscler Thromb Vasc Biol 34:742-750. doi:10.1161/ATVBAHA.113.301655 